\title{
Invited comment to the 'AbdoMAN': an artificial abdominal wall simulator for biomechanical studies on laparotomy closure techniques. L. F. Kroese, J. J. Harlaar, C. Ordrenneau, J. Verhelst, G. Guérin, F. Turquier, R. H. M. Goossens, G.-J. Kleinrensink, J. Jeekel, J. F. Lange
}

\author{
C. Hollinsky ${ }^{1}$
}

Received: 6 June 2017 / Accepted: 24 August 2017 / Published online: 14 September 2017

(C) Springer-Verlag France SAS 2017

The 'AbdoMAN' artificial abdominal wall simulator is an interesting new tool for biomechanical studies on laparotomy closure techniques. This device showed repeatable results of strain patterns, but in this study no closure technique was tested up to failure and at the end it is impossible to say how close or far away from reality this artificial abdominal wall simulator is.

However, there are some inaccuracies in the model and test arrangements that may lead to misinterpretations. I will explain some of these concerns as follows:

- In the first video of this study, it is clearly shown that the forces act on the lateral edges of the artificial abdominal wall and this synthetic abdominal wall gets lengthened badly close to the acting force. Only unknown fewer forces act at the midline, at the point where the measureable load should be.

- A further difference from reality is that only horizontal forces act. In reality, vertical loads are around 50\% compared to horizontal loads, but in this artificial abdominal wall simulator, no vertical loads act on the midline.

- In this setting, the authors simulate the intraabdominal pressure, because they think it is an important factor for the appearance of midline hernias after laparotomies. In reality,

This comment refers to the article available at doi:10.1007/ s10029-017-1615-x.

C. Hollinsky

christian.hollinsky@wienkav.at

1 Chirurgische Abteilung, Sozialmedizinisches Zenztrum Floridsdorf Krankenhaus und Geriatriezentrum, Hinaysgasse 1, 1210 Vienna, Austria the intraabdominal pressure is only a result of abdominal muscle contraction and has low influence on the incidence of abdominal wall hernias. But as a consequence of this setting, this $3.5 \mathrm{~L}$ air-filled Vacufix ${ }^{\circledR}$ collecting bag (used to represent the abdominal cavity) causes friction to the artificial abdominal wall and masks an unidentified high amount of force exerted on the midline.

- At least one of the main disadvantages of the 'AbdoMAN' compared to living tissue is that artificial or dead tissue (it is planned to take abdominal walls from human or animal cadavers for further studies) does not remodel. Hernias arise after months and years. In this time the fibers of the linea alba will get re-orientated due to the different loads to the midline. As a consequence to this, the comparisons between outcome using two different laparotomy closure techniques will be distinguishable first after this reorganization of living tissue over time. This cannot be shown with repeated tensile tests on dead or artificial tissue.

In conclusion, I would hesitate to use this new tool for biomechanical studies on laparotomy closure techniques.

Compliance with ethical standards

Conflict of interest The author declares that he has no conflict of interest.

Ethical approval This study design is confirmed to ethical standards.

Human and animal rights This article does not contain any studies with animals performed by the author.

Informed consent There were no individuals participants included in the study. 